\title{
Exposición espontánea de un torus palatino de la línea media
}

\author{
Gustavo Sinisterra, Jaime Álvarez, Pablo Emilio Molano \\ Escuela de Odontología, Universidad del Valle, Cali, Colombia
}

\begin{abstract}
Introducción. El torus, o rodete, palatino es una alteración ósea benigna que puede generar molestias en la fonación y en la deglución. Cuando su crecimiento o exposición persistente producen síntomas molestos, debe ser resecado quirúrgicamente.

Presentación del caso. Se trata de una paciente de 82 años de edad, que consultó por una lesión ulcerativa de un año de evolución, que producía dolor, halitosis y molestia a la deglución.

En el examen físico de la cavidad oral, se observó una prominencia de consistencia dura en la línea media, con perforación y ulceración de la mucosa palatina en su porción lateral posterior izquierda. Se consideraron los diagnósticos de torus palatino, osteoma, granuloma piógeno y neoplasia de tejidos blandos. Se decidió resecarla quirúrgicamente y hacer el estudio histopatológico. La evolución clínica fue satisfactoria, con resolución completa un mes después de la cirugía.

En el estudio de histopatología se observó hiperostosis ósea con inflamación crónica de los tejidos blandos e hiperplasia simple de la mucosa, que corresponden a un torus palatino.

Discusión. Se reporta un inusual caso de exposición espontánea de un torus palatino que ocupaba casi toda la bóveda palatina, se describe su resección quirúrgica y se informan los hallazgos de histopatología. En la revisión bibliográfica realizada, no se encontró un reporte previo de exposición espontánea de torus palatino.
\end{abstract}

Palabras clave: paladar, hiperplasia, exostosis, hiperostosis.

doi: http://dx.doi.org/10.7705/biomedica.v33i1.1480

\section{Spontaneous exposition of a midline palatal torus}

Introduction: Palatal torus, or torus palatinus, is a benign bone alteration that may cause some discomfort during phonation or swallowing. When its growth or persistent exposition produces unpleasant symptoms, it must be surgically removed.

Case presentation: We treated an 82-year-old female patient who consulted for a painful ulcerous lesion she had had for a year and which produced halitosis and discomfort when swallowing.

During the oral cavity physical exam we observed a hard protuberance on the midline. It showed a perforation and ulceration of the lateral posterior palatal mucosa on the left side. We considered the following diagnosis: palatal torus, osteoma, pyogenic granuloma, or a soft-tissue neoplasia. We decided to surgically remove it and to perform a histopathological examination. Clinical evolution was satisfactory with complete resolution one month after surgery.

The histopathological examination showed hyperostosis along with chronic inflammation of the soft tissues and a simple hyperplasia of the mucosa, which in turn indicated a palatal torus.

Discussion: We report an unusual case of spontaneous exposition of a palatal torus which took up almost all of the hard palate area. Its resection is described and we inform the histopathological findings. In the literature review, we did not find a previous report of a spontaneous exposition of a palatal torus.

Key words: palate, hyperplasia, exostoses, hyperostosis.

doi: http://dx.doi.org/10.7705/biomedica.v33i1.1480

Correspondencia:

Pablo Emilio Molano, Calle $5 \mathrm{~N}^{\circ}$ 38-25, consultorio 408, Edificio

Plaza San Fernando, Cali, Colombia.

Teléfonos: (572) 5588812 y (310) 4125818

pablomol42@hotmail.com

Recibido: 05/05/11; aceptado:06/09/12

Contribución de los autores:

Gustavo Sinisterra hizo el examen inicial del paciente y el diagnóstico, tomó las fotografías clínicas y elaboró la placa de protección palatina.

Jaime Álvarez practicó el procedimiento quirúrgico.

Pablo Molano prestó la ayudantía quirúrgica, hizo la toma y el envío de las muestras, y se encargó del control posoperatorio de la paciente; además, escribió el manuscrito para la revisión y aprobación de los otros dos autores.
La hiperostosis se define como la neoformación de carácter benigno del tejido óseo o la hipertrofia difusa o localizada del mismo. En la cavidad oral puede afectar la superficie lingual de la mandíbula (torus mandibularis) o del paladar duro (torus palatinus) o presentarse en forma de exostosis de localización

paciente; además, escribió el manuscrito para la revisión y aprobación de los otros dos autores. 
múltiple. Clínicamente, se manifiesta como prominencias compactas recubiertas por mucosa de aspecto sano, generalmente, asintomáticas.

Los nombres de "torus palatino" y "torus lingual" fueron introducidos por Dupfer y Besselhagen en 1897, el primero, para describir la excrecencia ósea en la región palatina media y, el segundo, aquella en la superficie lingual de la mandíbula en la zona de los premolares y el primer molar inferior (1).

Varios autores, como Reichart (2), Kerpond y Sirirungrojying (3) y Chohayed (4), reportaron el predominio del torus, o rodete, palatino frente al mandibular. No obstante, otros, como Bruce, et al. (5), y Al Quran y Al-Dwairi (6), encontraron una mayor prevalencia del torus mandibular que del palatino, sin poder explicar esta discrepancia. Por otra parte, Axelsson y Ellegard (7) y Karaiscaraiscos, et al. (8), reportaron igual frecuencia en los dos tipos. La prevalencia del torus palatino está entre 6 y $8 \%$ en los Estados Unidos y llega hasta $25 \%$ en otras poblaciones del mundo (9-12).

El desarrollo del torus mandibular parece estar relacionado con el número de dientes presentes $y$ funcionales, y, en muchas ocasiones, se ha asociado con bruxismo (13), factores hereditarios y del medio ambiente, ingestión de medicamentos (1) o un patrón de herencia familiar.

Con menor frecuencia, se encuentran otros tipos de exostosis que afectan los maxilares, especialmente en el aspecto bucal de los alvéolos maxilares y en otros sitios atípicos, y que comúnmente son provocados por trauma, reacción perióstica, inflamación o neoplasia $(14,15)$.

Los tori son de crecimiento lento a lo largo de la vida, más prevalentes entre los 11 y los 30 años de edad, y de muy rara aparición antes de los 10 años. Se estima que $10 \%$ de la población puede verse afectada y es más frecuente en mujeres que en hombres (16).

Según Eggen y Natvug, son más frecuentes entre los 10 y los 49 años, y de rara aparición después de los 50 (17).

Según su forma, los tori palatinos se dividen en planos, unilobulados, multilobulados, nodulares, irregulares o fusiformes; esta última es la más común $(18,19)$.

En la histopatología, el torus se caracteriza por excrecencia de tejido óseo denso con un patrón laminado y espacios pequeños ocupados por médula ósea gruesa o estroma fibrovascular esparcido, en donde se puede observar mínima actividad osteoblástica u, ocasionalmente, importante actividad perióstica (20).

Los tori requieren tratamiento cuando son grandes, alteran la función, generan desplazamiento dental o producen trauma y ulceración de la superficie mucosa, pero, en especial, cuando imposibilitan la colocación y uso de las prótesis totales o removibles.

Cuando el tratamiento está indicado, las lesiones pueden ser talladas o removidas quirúrgicamente, cortándose desde su base de unión (1).

La exposición espontánea de los tori es un fenómeno poco común y se ha considerado como un secuestro óseo. Se clasifica como primario, si se mantiene enteramente separado, como secundario, si está parcialmente separado, y como terciario, si está separado solo por una leve línea divisoria y se mantiene en su lugar (21). La exposición de un torus y el subsecuente secuestro óseo tienen múltiples causas, como son las proyecciones agudas del torus que causan irritación crónica por daño traumático producido por alimentos duros, el cepillado o hábitos orales, posible constricción vascular, exceso de fuerzas de oclusión o trauma quirúrgico (22).

Con el fin de documentar de mejor forma este caso, se hizo una revisión detallada de las siguientes bases de datos: Cochrane Oral Health Group, Cochrane Central Register of Controlled Trials (CENTRAL), Cochrane Library 2010, Medline (1950 a junio de 2010), Embase (1980 a 2 de junio de 2010); además, de las revistas Journal of Periodontology, Journal of Clinical Periodontology, Periodontology 2000 e International Journal of Periodontal and Restaurative Dentistry.

El propósito del presente artículo fue reportar un caso de exposición espontánea de un torus palatino que ulceró la mucosa, por lo cual tuvo que ser removido quirúrgicamente, y se exponen la evaluación clínica y el resultado del estudio histopatológico del tejido palatino retirado.

\section{Presentación del caso}

Se trata de una mujer de 82 años de edad, con $45 \mathrm{~kg}$ de peso, que consultó por presentar una ulceración persistente en el paladar duro que producía intenso dolor, halitosis y dificultad para deglutir. La paciente refería la presencia de una masa palatina de 60 años de evolución con ulceración en el último año y que en los últimos dos 
meses había tenido un crecimiento exagerado, lo que aumentó las molestias descritas.

En el examen clínico de la cavidad oral, se encontraron 28 piezas dentales en total, con incrustaciones en oro y coronas de metal-porcelana en los molares y premolares. El periodonto estaba sano, sin bolsas ni inflamación gingival.

En el paladar se observó una masa de consistencia firme en la línea media, que medía $5 \times 3 \times 1,5 \mathrm{~cm}$, aproximadamente, y ocupaba casi toda la bóveda palatina. La mucosa que la cubría era muy delgada y, en el lado izquierdo, mostraba una zona de ulceración de un diámetro aproximado de $7 \mathrm{~mm}$ (figura 1).

Por su apariencia clínica, se consideró el diagnóstico diferencial entre torus palatino expuesto y una lesión tumoral de tipo osteoma, si su origen fuera de tejido óseo, o, en caso de originarse en los tejidos blandos, entre un granuloma piógeno y una neoplasia. Por esta razón, se decidió practicar resección quirúrgica de la lesión.

La paciente firmó el consentimiento informado para el procedimiento quirúrgico que se iba a practicar, y autorizó la evaluación histopatológica y la publicación del caso clínico.

En el periodo preoperatorio, se practicaron los exámenes de laboratorio de rigor y la valoración por anestesiología general; el caso se manejó con fase higiénica periodontal, control de la placa bacteriana y refuerzo de las medidas de higiene oral.

Se tomaron impresiones del arco superior e inferior, las que se enviaron al laboratorio dental para la confección de una placa palatina en acrílico, que se utilizó como soporte y protección después del procedimiento quirúrgico.

Se planeó la extirpación del torus palatino, haciendo uso de estrictas medidas de asepsia y bajo anestesia general. Se inició con una incisión en el rafe palatino a lo largo de toda la extensión de la lesión. Se disecaron y elevaron colgajos mucoperiósticos laterales completos, hasta llegar al paladar óseo. Se practicó osteotomía con una fresa refrigerada de baja velocidad en los surcos anteriores y laterales de la lesión, con una profundidad de casi un centímetro de profundidad. La osteotomía se continuó con cincel, allanando la superficie ósea, hasta reconstruir la anatomía de la bóveda palatina con una fresa redonda para hueso. Luego, se hizo la plastia de los tejidos blandos, adaptando los colgajos y suturando con puntos simples. Finalmente, se colocó la placa acrílica con

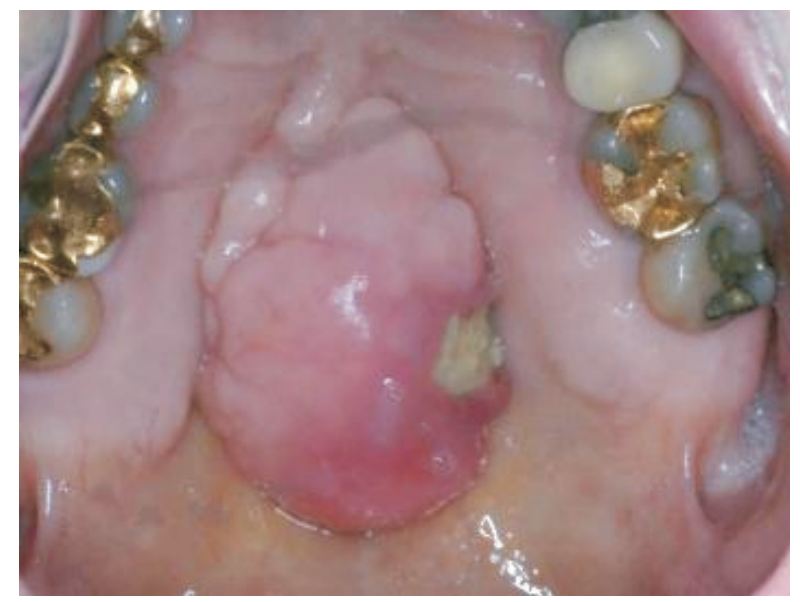

Figura 1. Exposición espontanea de torus palatino. Se aprecia masa multilobulada que ocupa la zona central de la bóveda palatina, con cambios en la mucosa que la recubre y zona de ulceración con inflamación circundante en su aspecto izquierdo y posterior.

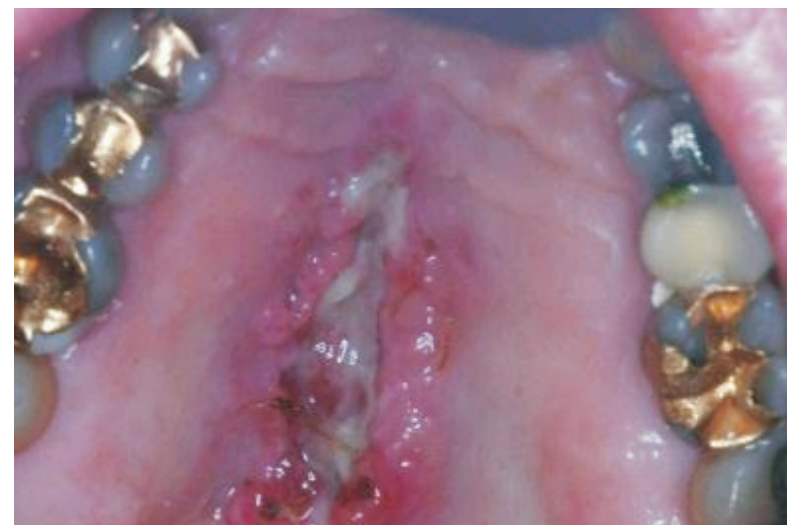

Figura 2. Fase de cicatrización 12 días después de la extirpación de la lesión.

pasta Coe-Flex ${ }^{T M}$ sobre toda la superficie palatina, la cual protegía la herida, prevenía la hemorragia o formación de hematomas, la protegía contra el trauma por alimentos y ayudaba a la cicatrización.

Una semana más tarde se hizo el control posoperatorio y se colocó nuevamente la placa con Coe-Flex ${ }^{\mathrm{TM}}$. Doce días después se retiraron la placa y los puntos de sutura, y se limpió la herida (figura 2). A los seis meses, se comprobó una evolución clínica satisfactoria, con aspecto normal del paladar (figura 3).

Para el estudio de histopatología se remitieron cuatro fragmentos de menos de 0,6 $\times 0,3 \mathrm{~cm}$ de tejido epitelial erosionado, seis fragmentos de 1,5 $\mathrm{cm}$ de tejido óseo de aspecto sano y tres fragmentos de $1 \mathrm{~cm}$ de hueso del área necrótica.

En la mucosa oral, se encontró notable hiperplasia reactiva del epitelio escamo-celular con 


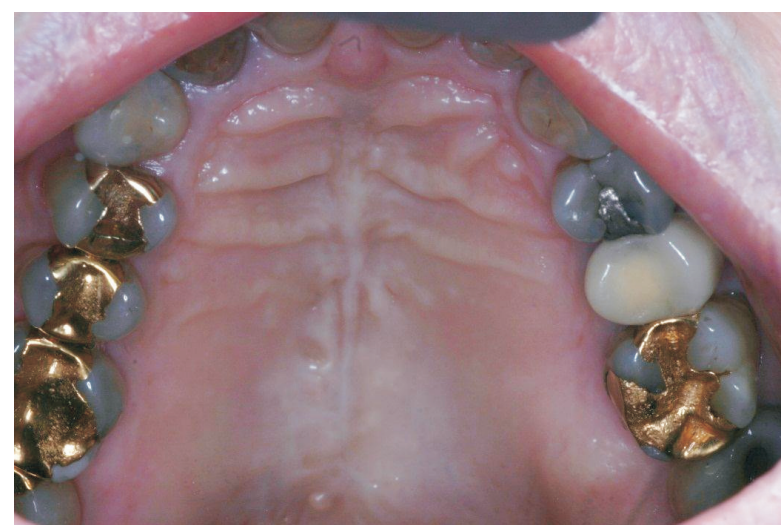

Figura 3. Evaluacion clinica a los 6 meses. El paladar presentaba un aspecto sano.

paraqueratosis sin atipias nucleares en la dermis; además, extensos infiltrados inflamatorios que, en algunos sitios, eran de tipo crónico y linfoplasmocitario $y$, en otros, se asociaban con neutrófilos polimorfonucleares que formaban microabscesos. Todo esto demostraba un importante proceso inflamatorio activo, posiblemente debido a ulceración por trauma local (figura 4).

El tejido óseo presentaba cambios de hiperostosis, consistentes en aumento del grosor de la capa de hueso compacto y de la densidad del hueso trabecular, sin ninguna evidencia de transformación maligna.

Se hizo diagnóstico histopatológico de hiperplasia simple de la mucosa, con proceso inflamatorio crónico e importante actividad aguda de vecindad, e hiperostosis ósea (torus palatinus) (figura 5).

\section{Discusión}

Teniendo en cuenta que en la revisión bibliográfica llevada a cabo no se encontró ningún reporte previo de exposición espontánea de torus palatino con ulceración crónica de la mucosa palatina, se decidió publicar este caso para ilustración de la comunidad científica.

En 1997, Sonnier y Horning (21) reportaron cuatro casos de torus mandibular lingual en los que hubo exposición espontánea y secuestro del hueso alveolar, que se sometieron a cirugía y de los cuales no se informaron los resultados del estudio de histopatología. Uno de ellos se asoció con mucositis alérgica por prótesis. En los otros tres, la exostosis era de tipo idiopático y se caracterizó por presentar proyecciones agudas y rugosas, las cuales fueron removidas quirúrgicamente sin que se presentara recurrencia de las lesiones.

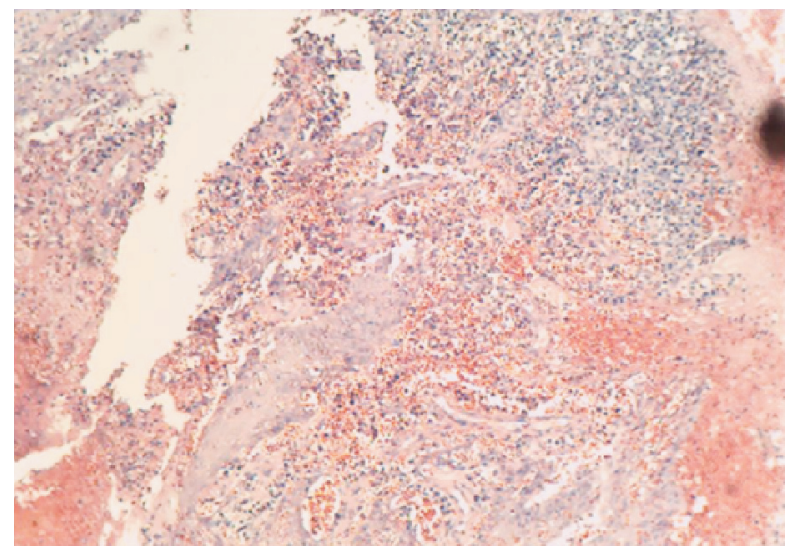

Figura 4. En el tejido palatino blando se aprecia ulceración epitelial, hiperplasia reactiva, paraqueratosis, infiltrado inflamatorio y ausencia de atipias nucleares en el corion. Hematoxilina y eosina, 100X.

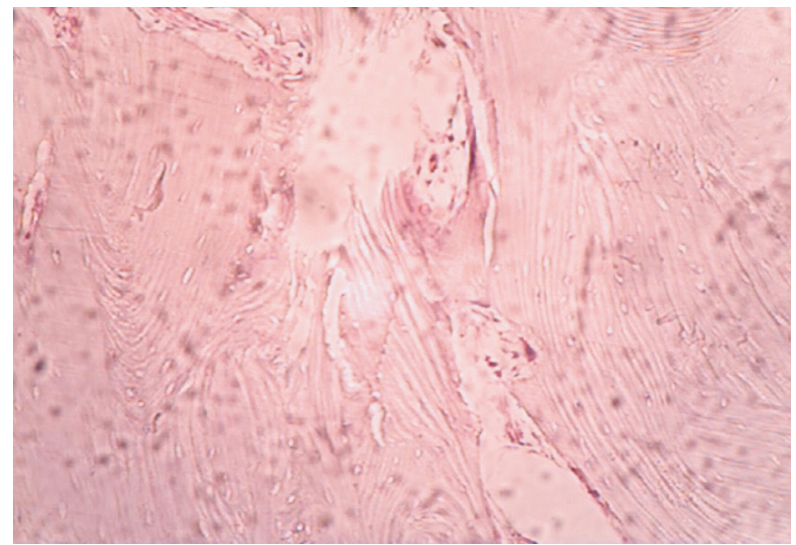

Figura 5. En el tejido palatino duro se observa hiperostosis palatina caracterizada por aumento del grosor de la capa de hueso compacto y de la densidad del hueso trabecular sin evidencia de un proceso maligno. Hematoxilina y eosina, 400X.

En el caso presentado, no se encontró asociado ninguno de los factores antes mencionados, como dentición, bruxismo, herencia, medio ambiente, etc., lo que nos permite suponer que su origen fue idiopático.

En conclusión, la exposición espontánea de un torus palatino es un fenómeno poco común que al no resolverse puede generar problemas en la fonación, la deglución y la función masticatoria, por lo cual está indicado practicar la escisión quirúrgica y la evaluación histopatológica para lograr el diagnóstico y tratamiento adecuados.

\section{Agradecimientos}

Los autores agradecen a la Escuela de Odontología de la Universidad del Valle, por permitir la realización del procedimiento quirúrgico. 


\section{Conflicto de intereses}

No existe ninguno.

\section{Financiación}

El procedimiento quirúrgico y la evaluación histopatológica fueron realizados y subvencionados por los autores.

\section{Referencias}

1. Manotas I, Pertus VL, Escorcia LS. Torus palatino, torus mandibular y exostosis mandibulares. Revista de la Facultad de Ciencias de la Salud. 2005;2:115-23.

2. Reichart PA, Neuhaus T, Sookasem M. Prevalence of torus palatinus and torus mandibularis in Germans and Thai. Community Dent Oral Epidemiol. 1988;16:61-4.

3. Kerdpon D, Sirirungrojying S. A clinical study of oral tori in southern Thailand: Prevalence and the relation to parafunctional activity. Eur J Oral Sci. 1999;107:9-13. http:// dx.doi.org/10.1046/j.0909-8836.1999.eos107103.x

4. Chohayeb AA, Volpe AR. Ocurrence of torus palatinus and mandibularis among women of different ethnic groups. Am J Dent. 2001;14:270-80.

5. Bruce I, Ndau TA, Addo ME. Epidemilogical aspects of oral tori in a Ghanaian community. Int Dent J. 2004;54:78-82.

6. Al Quaran FA, Al-Dwairi ZN. Torus palatinus and torus mandibularis in edentulous patients. J Contemp Dent Pract. 2006;7:112-9.

7. Axelsson G, Ellegard B. Torus mandibularis among Icelanders. Am J Phys Anthropol. 1981;54:383-9. http:// dx.doi.org/10.1002/ajpa.1330540312

8. Karaiscaraiscos S, Dimitriou P, Tsironis G, Spyropoulos ND. Un estudio clínico y epidemiológico de los torus mandibulares. Odontostomatol Proodos. 1989;43:443-9.

9. King DR, King AC. Incidence of tori in three population groups. J Oral Med. 1981;36:21-3.

10. King DR, Moore GE. An analysis of torus palatinus in a transatlantic study. J Oral Med. 1976;31:44-6.
11. Eggen S, Natvig B. Relationship between torus mandibularis and number of teeth present. Scand J Dent Res. 1986;94:233-40. http://dx.doi.org/10.1111/j.16000722.1986.tb01758.x

12. Suzuki M, Sakai T. A familial study of torus palatinus and torus mandibularis. Am J Phys Anthropol. 1960;18:263-72. http://dx.doi.org/10.1002/ajpa.1330180404

13. Shafer WG, Hine MK, Levy BM. A textbook of oral pathology. 4th edition. Philadelphia: W. B. Saunders Company; 1983. p. 167-9.

14. Seah YH. Torus palatinus and torus mandibularis: A review of the literature. Aust Dent J. 1995; 40:318-21. http://dx.doi. org/10.1111/j.1834-7819.1995.tb04820.x

15. Antomiades DZ, Belozi M, Papanayiotou P. Concurrence of torus palatinus with palatal and buccal exostosis: Case report and review of the literature. Oral Surg Med Oral Pathol Oral Radial Endod. 1998;85:552-7. http://dx.doi. org/10.1016/S1079-2104(98)90290-6

16. Shah DS, Sanghavi SJ, Chawda JD, Shah RM. Prevalence of torus palatinus and torus mandibularis in 1,000 patients. Indian J Dent Res. 1992;3:107-10.

17. Eggen S, Natvig B. Relationship between torus mandibularis and number of present teeth. Scand J Dent Res. 1986;94:233-40

18. Kolas S, Halperin V, Jefferis K. The occurrence of torus palatinus and torus mandibularis in 2,478 dental patients. Oral Surg Oral Med Oral Pathol 1953;11:1134-8. http:// dx.doi.org/10.1016/0030-4220(53)90225-4

19. Flynn MW, Martínez NP, Meyer CJ. Torus palatinus. Report of a case. Am J Dent. 1992;5:339-42.

20. Johnson OM. The tori and masticatory stress. J Prosthetic Dent. 1959;9:975-7.

21. Sonnier KE, Horning GM. Spontaneous bony exposure: A report of 4 cases of idiopathic exposure and sequestration of alveolar bone. J Periodontol. 1997;68:758-62.

22. Krolls SO, McGinnis JP. Exophytic hard tissue mass: A case report. Dentistry. 1992; 12:19-20. 